\title{
Association of Genetic Variation in the Promoter Region of OXTR with Differences in Social Affective Neural Processing
}

\author{
Garret O'Connell $^{1}$, Heather C. Whalley ${ }^{1}$, Prerona Mukherjee ${ }^{1}$, Andrew C. Stanfield ${ }^{1}$, \\ Christian Montag ${ }^{2}$, Jeremy Hall ${ }^{1}$, Martin Reuter ${ }^{2,3}$ \\ ${ }^{1}$ Division of Psychiatry, University of Edinburgh, Scotland, UK \\ ${ }^{2}$ Department of Psychology, University of Bonn, Bonn, Germany \\ ${ }^{3}$ Center for Economics and Neuroscience, University of Bonn, Bonn, Germany \\ Email: G.OConnell@pgr.reading.ac.uk
}

Received September 8, 2011; revised October 13, 2011; accepted November 11, 2011

\begin{abstract}
Evidence supports the involvement of oxytocin in social behavior. The oxytocin receptor gene (OXTR) has been associated with differences in social brain function and risk for autism. Motivated by recent work, we investigated the effect of variation in the common functional rs2268498 T/C polymorphism in the promoter region of OXTR on neural responses to fear expressions. 46 healthy subjects were divided into genotype groups of C carriers $(n=32)$ and TT homozygous $(n=14)$ and neural activity was measured during the recognition of fear and neutral expressions. Results showed that during the recognition of fear expressions, the TT genotype group exhibited increased responding in the inferior occipital gyrus, considered important for face processing, compared to carriers of the $\mathrm{C}$ allele $(P<0.005$; cluster corrected for whole brain), an effect not found for neutral faces. These results indicate the impact of this OXTR genetic variant on individual differences in social affective neural processing.
\end{abstract}

Keywords: OXTR; Promoter Region; Inferior Occipital Gyrus; fMRI; Emotional Facial Processing

\section{Introduction}

The abundant neuropeptide oxytocin is an essential regulator of social behaviour. Initial evidence for this role came from animal research reports that oxytocin promotes maternal bonding [1], mate affiliation and social recognition [2]. Synthesized in the hypothalamus, oxytocin is released in limbic brain areas important for social cognition [3]. Regional expression of oxytocin has been found to explain differences in sociality within and between species [2].

In line with its prosocial effects in animals, oxytocin has been shown to enhance trust [4] and generosity in humans [5]. Evidence has gathered to suggest that oxytocin exerts its prosocial effects by regulating the aversiveness of social experiences, such as reducing the impact of betrayal on trust behaviour [4,6]. A recent important study shows that oxytocin also produces non-prosocial effects, such as increased gloating and envy [7]. Based on this evidence, the effect of oxytocin has been reinterpreted as the enhanced motivation for all types of social approach behaviours by inhibiting fear responses to social stimuli [8].

A social approach behaviour for which the effects of oxytocin have been intensively studied is emotion recognition of facial expressions. Systematically infused oxytocin has been shown to improve the accuracy of emotion recognition [9-11]. Moreover, studies report that oxytocin alleviates emotion recognition deficits among persons with autism spectrum conditions (ASC) [12,13], generating much clinical interest in the neuropeptide [14]. Hence, accumulating evidence suggests that oxytocin enhances the perception of non-verbal social information such as facial expressions.

An emerging account suggests that oxytocin enhances emotion recognition by altering emotional evaluations of facial expressions. It has been shown that expressions interpreted as threatening lead persons to avert their gaze from emotionally salient eye regions [15,16]. Evidence indicates that oxytocin reduces the aversiveness of faces $[17,18]$. Eye-tracking data also indicates that oxytocin increases gaze to eye regions [19]. Moreover, oxytocin has been found to ameliorate eye-fixation deficits in persons with ASC [20,21]. Hence, oxytocin may facilitate social communication by overcoming avoidance reactions and enabling increased depth of processing of facial affect. Evidence that oxytocin-enhanced emotion recognition is 
strongest for threatening expressions support this view [22,23].

Investigations using functional magnetic resonance imaging (fMRI) provide further evidence that oxytocin modulates social approach by regulating neural adaptations to social fear. Reduced responding in neural bases of social fear such as the amygdala is shown to be concurrent with oxytocin-induced increases in prosocial behaviour [24]. The amygdala is a brain structure abundant in oxytocin receptors [25] that underlies the processing of fearful [26] and social stimuli $[27,28]$, and which coordinates activity in other social brain regions. One expression of amygdala arousal to perceived threat is enhanced activity in the fusiform and inferior occipital gyrus (IOG) [29]. In sum, these findings suggest that oxytocin enhances social approach by reducing neural responses to social fear.

Compared to prosocial behaviour, the neural impact of oxytocin during emotion recognition is less clear. In line with prosocial behaviour, some evidence indicates that activation in the aforementioned brain regions in response to aversive social stimuli is attenuated by oxytocin [30,31]. In contrast, enhanced amygdala responses to facial expressions (regardless of valence) following oxytocin administration have also been reported [32]. It has been suggested that attentional artefacts might explain the inconsistency in these findings [33].

Gamer and colleagues [34] used combined fMRI and eye-tracking to investigate the link between social attention and the neural impact of oxytocin. While they reported oxytocin overall attenuated amygdala activity to fear expressions, they found that during eye gaze fixation periods there was an increase in amygdala activation similar to levels characteristic of processing "happy" faces. Hence, oxytocin has opposite effects on amygdala activation depending on whether attention is directed to facial affect. This might facilitate greater depth of emotion processing by reducing neural fear responding when facial affect is not being attended to, leading to subdued threat avoidance reactions, and finally, increased attention to and neural processing of facial affect. In summary, oxytocin appears to enhance the social approach behaviour of emotion recognition, resulting in increased responding to social fear at the neural level.

An important determinant of oxytocin signalling is the availability of receptor sites, coded for in humans by the oxytocin receptor gene (OXTR) [35]. Independent studies have reported evidence that variation in OXTR increases susceptibility for ASC [36-41], although there has been some heterogeneity in these findings [42]. Persons diagnosed with ASC often exhibit gross impairments in social functioning [43]. Furthermore, OXTR risk variants for ASC have been associated with individual differences in healthy populations in social behaviours of trust [42] and empathy [44,45]. Evidence therefore demonstrates the social importance of genetic variation in OXTR.

On the basis of this evidence, Tost et al. [46] investigated the impact of genetic variation in OXTR on the neural processing of threatening faces in healthy subjects. Amygdala underactivation was found to be associated with a putative ASC risk variant at the rs53576 single nucleotide polymorphism (SNP). Another risk variant at rs2254298 has been associated with smaller amygdala volume $[47,48]$. The summarized view of the discussed findings is that investigating the impact of genetic variation in OXTR on brain function during emotion recognition might help elucidate the psychophysiological role of oxytocin in social behaviour.

Recent evidence suggests that a T/C SNP at rs2268498, unexplored for neural effects and risk for ASC, may confer changes in social behaviour. Evidence from Reuter et al. (unpublished) found associations of the TT genotype with increased trust, and in another study with personality dimensions of negative emotionality [49]. However, the effect of genetic variation of the rs2268498 SNP on social brain function remains to be clarified.

In the current study, we examined the impact of allelic differences in the rs2268498 SNP on emotion processing among healthy individuals during fMRI scanning. We used the recognition of fear expressions condition that is known to robustly elicit activation differences in neural affective processing between healthy groups and persons with ASC. We hypothesize that if genetic variation in the rs2268498 polymorphism modulates oxytocin signalling during social fear responses, then the TT genotype group would exhibit reduced threat avoidance, and as a result, increased neural responses to fear expressions in comparison to $\mathrm{C}$ allele carriers.

\section{Methods}

\subsection{Participants}

Forty-six healthy Caucasian subjects participated in the study $($ male $=24$, female $=22$; mean age $=32.2$ ). All subjects were screened for and cleared of neurological, psychiatric, or substance-abuse problems and had no history of other medical problems or treatment relevant to cerebral metabolism and blood flow.

\subsection{Demographic and Genotype Data}

Prior to scanning, participants completed the National Adult Reading Test (NART) (Nelson, 1991) to control for variability in cognitive ability during analysis. All participants gave informed consent to take part in the study. Genomic DNA was extracted from venous blood samples. The genotyping was conducted by the Wellcome Trust Clinical Research Facility in Edinburgh (United Kingdom) and used standard TaqMan assays, by 
the TaqMan polymerase chain reaction based method (TaqMan, AssayByDesign, Applied Biosystems, Foster City, California). Genotyping was performed for one SNP from the OXTR complex (rs2268498).

\section{3. fMRI Data Acquisition}

Imaging was carried out on a GE 1.5 T Signa scanner (99 volumes; Field of View $22 \mathrm{~cm}$; Time to Echo (TE) $40 \mathrm{~ms}$; Volume acquisition time (TR) 2.5s) (GE Medical, Milwaukee, USA). Interleaved axial slices were acquired with a thickness of $5 \mathrm{~mm}$ with no gap and a matrix size of $64 \times 64$, and the first four volumes were discarded to avoid equilibrium effects. For the functional scan the parameters were (99 volumes TR/TE $=2500 / 40 \mathrm{~ms}$; field of view $(\mathrm{fov})=22 \mathrm{~cm}$ ). Visual stimuli were presented using a screen (IFIS, MRI Devices, Waukesha, WI, USA) placed in the bore of the magnet; corrective lenses were used where necessary. The T1 sequence yielded 180 contiguous $1.2 \mathrm{~mm}$ coronal slices $($ matrix $=192 \times 192$; fov $=24$ cm; flip angle $8^{\circ}$ ).

For each subject, echo planar image volumes were realigned to the mean volume. The images were then normalized to a study-specific template with a linear affine transformation, followed by non-linear deformations and resampled with sinc interpolation to cubic voxels of size $8 \mathrm{~mm}^{3}$. Normalized images were spatially smoothed with a 6-mm fullwidth at half maximum (FWHM) kernel to minimize residual inter-subject differences and meet assumptions for statistical analysis.

\section{4. fMRI Analyses}

Statistical analysis was performed using the general linear model approach as implemented in SPM2 (Wellcome Department of Cognitive Neurology and collaborators, Institute of Neurology, London, United Kingdom), running in Matlab (The MathWorks, Natick, MA, USA). Although we had a priori hypotheses about oxytocin's effects within social brain regions (e.g. amygdala, fusiform and IOG), to obviate bias and generate exploratory findings for subsequent hypotheses, we conducted a wholebrain voxel-wise analysis of variance (ANOVA) to examine the effect of genotype. To control for potential confounding of genotype effects by gender, we performed an additional SPM analysis using a multiple regression with genotype and sex as independent variables.. Images were thresholded at 0.005 uncorrected and whole brain cluster corrections for multiple comparisons were performed with only clusters at $P_{\text {corr }}<0.05$ considered significant.

A block design was used with three conditions: fear, neutral, and baseline (fixation). During fear blocks, six faces from the Ekman and Friesen series expressing fear were each presented for $3.5 \mathrm{sec}$ with a $0.5 \mathrm{~s}$ inter-stimulus interval; during neutral blocks, the same faces show- ing a neutral emotional expression were presented and instructions given to judge the gender of the person were given. During baseline blocks, participants were instructed to look at a fixation cross. Analysis was conducted to compare the neural responses to fearful faces with that to neutral faces (fear > neutral contrast). In addition separate contrasts were conducted to assess neural responses to fearful and neutral faces relate to the baseline condition (fear $>$ baseline and neutral $>$ baseline contrasts).

\section{Results}

\subsection{Genotype Group Demographic and Behavioural Differences}

Based on the genotype data for the rs2268498 SNP, individuals possessing either one $(n=24)$ or two copies ( $n$ $=8)$ of the $\mathrm{C}$ allele were included in the C-carriers group ( $n=32)$, and those homozygous for the T allele $(n=14)$ were included in the T/T group. Genotypes were in Hardy-Weinberg Equilibrium ( $\mathrm{Chi}^{2}=3.17$, n.s.). There were no significant differences between the groups in terms of age, NART, gender, years of education or performance on emotion recognition tasks $(p<0.05)$. This SNP was low in linkage disequilibrium with the rs53576 $\left(D^{\prime}=0.53\right)$ and rs2254298 $\left(D^{\prime}=0.62\right)$, previously implicated SNPs on brain function and risk for ASC (LD scores estimated using haploview software [50]).

\subsection{Genotype Group Differences in Brain Activity}

The subjects demonstrated the expected patterns of brain activation and behavioural responses indicating subjects were performing the task correctly in the scanner (Figure 1). Activation during fear versus baseline included bilateral lateral and medial prefrontal regions, medial temporal lobe, sub-cortical structures, parietal cortex and occipital regions $\left(P_{\text {corr }}<0.01\right)$. For fear versus neutral activated regions were restricted to the right inferior frontal gyrus, left amygdala, and right posterior superior temporal gyrus and fusiform gyrus.

Genetic imaging analysis revealed several suprathreshold clusters (for a summary of results, see Table 1). Most notably, significantly greater bilateral IOG activity was found in the TT group versus $\mathrm{C}$ carriers in the contrast of fear $>$ neutral (Figure 2). No gender differences were reported in this effect. This effect was non-significant in the contrast of neutral faces > baseline, indicating that the effect of variation in OXTR is highly specific to the emotional component of the task and was not a general effect of face processing.

\section{Discussion}

In the current study we found that genetic variation in the 

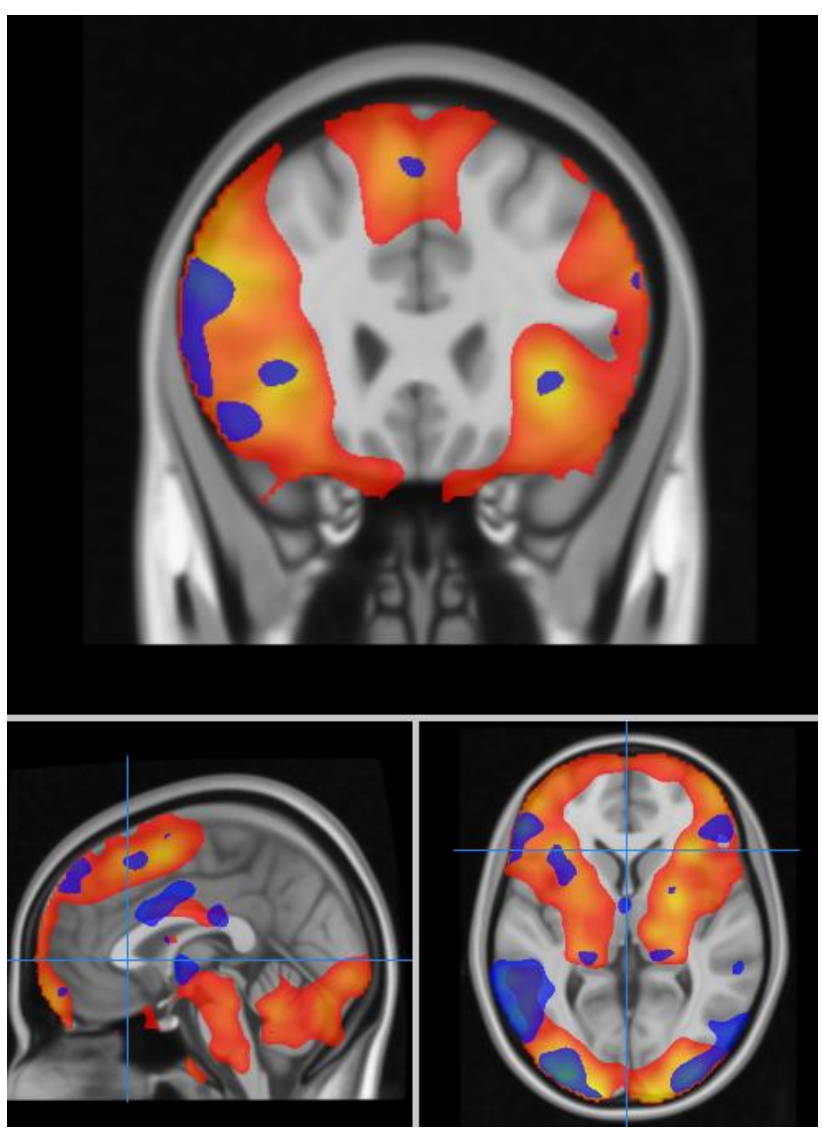

Figure 1. Task related brain activation for an emotion processing task (red colour-scale represents contrast of fear versus baseline, blue colour-scale represents fear versus neutral discrimination).

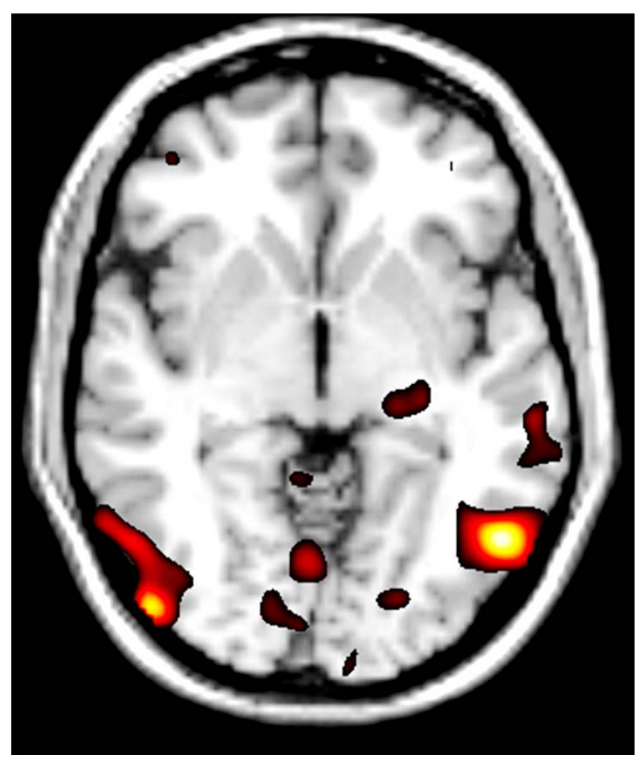

Figure 2. Effect of OXTR genetic variation at SNP rs2268498 during the emotion processing task. The $\mathrm{T} / \mathrm{T}$ group $(n=14)$ had bilaterally increased activity of the IOG compared to C Carriers $(n=32)$ [Peak coordinates, Left: $x=$ $-52, y=-62, z=-4$; Right: $x=48, y=-82, z=-2$ ].
Table 1. Random effects analysis: main group differences.

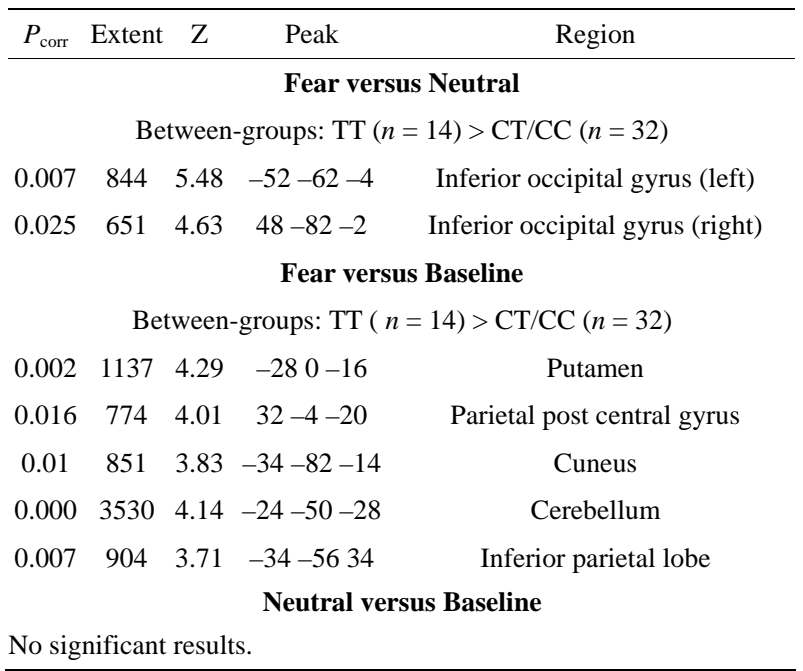

rs2246898 SNP in OXTR, a gene implicated in social behaviour and for which the neural effects have not yet been explored, was associated with alterations in social brain function. The results showed that during the processing of fear expressions, those homozygous for the $\mathrm{T}$ allele demonstrated relatively increased activation in the IOG. This suggests the importance of genetic variation in OXTR to individual differences in the processing of social affective information. Views on the neuromodulatory role of the amygdala during emotion processing give context to the current findings.

The amygdala processes the valence of faces [51] and signals their emotional salience to other brain areas [52]. It has been suggested that IOG responses to facial valence is due to amplified inputs from the amygdala [29,30, 53]. Hence, the results reported here are consistent with previous findings of the modulating effect of exogenously administered oxytocin $[6,24,30]$ and genetic variation in OXTR [46] on emotion processing in the amygdala and connected social brain regions .

The C allele of the rs2268498 SNP has previously been associated with low scores on personality dimensions of trust and the predisposition for negative emotions [49]. In line with this, here we found an association of the $\mathrm{C}$ allele with underactivation in the IOG during the processing of fear expressions. In light of previous reports that exogenously administered oxytocin increases responses in this area to facial affect [32], these findings convergently suggest a negative association of the $\mathrm{C}$ allele with both oxytocin signaling and the depth of processing of social affective information. However, these results are preliminary and further studies are needed to examine the relationship of this polymorphism on oxytocin expression and receptor availability.

Activation in the amygdala, which modulates inputs into the IOG, is enhanced by oxytocin after controlling 
for attentional artefacts [34]. Crucially, responses in the IOG to fear expressions are also shown to be modulated by attention, suggesting that these results are driven by the effects of oxytocin on social approach behaviour (i.e., enhanced attention to and evaluation of facial affect) [54]. However, the lack of eye-tracking data precludes conclusions that these differences in emotional processing are mediated by genetic effects on threat avoidance reactions.

These effects of the rs2268498 SNP in OXTR on emotion processing in the IOG demonstrate some correspondence with aberrant social brain function in persons with ASC. Amygdala dependent activation, such as IOG responses to facial affect, is purportedly central to social perceptual deficits experienced by persons with ASC [55]. Indeed, IOG activation during facial processing is reported as abnormal in ASC [56]. These results suggest that a neural pathway involving the IOG and amygdala mediates the putative risk of genetic variation in OXTR for social deficits in ASC.

A foremost limitation of this study is the uncertainty surrounding the rs2268498 SNP in terms of genetic functionality. Another consideration neglected in this study is the sensitivity of the oxytocinergic system to environmental factors. Studies indicates that maternal caregiving in animals [57] and cultural differences in humans [58] modulate the impact of OXTR variation on social behaviour. Lastly, association with risk for ASC needs to be investigated to support claims of this SNP's involvement in the psychopathological deficits exhibited in persons with the disorder.

In summary, these results demonstrate the effect of genetic variation in a novel OXTR polymorphism on the neural processing of social affective cues. This parallels previous findings of the impact of oxytocin on the neural processing of threatening social signals. Lastly, the overlap of the neural impact of the rs2268498 variant in $O X$ $T R$ with aberrant brain function implicated in ASC warrants further investigation of the effect of this polymerphism in clinical populations.

\section{Acknowledgements}

Genotyping was performed at the Wellcome Trust Clinical Research Facility, Edinburgh. Brain Imaging was performed at the SFC Brain Imaging Research Centre in Edinburgh. We would like to thank all the participants. This study was funded by the Dr Mortimer and Theresa Sackler Foundation and was additionally funded by the German Research Foundation (RE-1692-4-1). ACS is supported by a Wellcome Trust Clinical Research Fellowship. JH is supported by a Scottish Senior Clinical Fellowship.

\section{REFERENCES}

[1] T. Insel and L. Shapiro, “Oxytocin Receptor Distribution
Reflects Social Organization in Monogamous and Polygamous Voles," Proceedings of the National Academy of Sciences USA, Vol. 89, No. 13, 1992, pp. 5981-5985. doi:10.1073/pnas.89.13.5981

[2] L. J. Young and Z. Wang, "The Neurobiology of Pair Bonding," Nature Neuroscience, Vol. 7, No. 10, 2004, pp. 1048-1054. doi:10.1038/nn1327

[3] R. Landgraf and I. D. Neumann, "Vasopressin and Oxytocin Release within the Brain: A Dynamic Concept of Multiple and Variable Modes of Neuropeptide Communication," Frontiers in Neuroendocrinology, Vol. 25, No. 3-4, 2004, pp. 150-176. doi:10.1016/j.yfrne.2004.05.001

[4] M. Kosfeld, M. Heinrichs, P. J. Zak, U. Fischbacher and E. Fehr, "Oxytocin Increases Trust in Humans," Nature, Vol. 435, No. 7042, 2005, pp. 673-676. doi:10.1038/nature03701

[5] P. Zak, A. A. Stanton and S. Ahmadi, "Oxytocin Increases Generosity in Humans,” PLoS ONE, Vol. 2, No. 11, 2007, p. e1128. doi:10.1371/journal.pone.0001128

[6] T. Baumgartner, M. Heinrichs, A. Vonlanthen, U. Fischbacher and E. Fehr, "Oxytocin Shapes the Neural Circuitry of Trust and Trust Adaptation in Humans," Neuron, Vol. 58, No. 4, 2008, pp. 639-650. doi:10.1016/j.neuron.2008.04.009

[7] S. G. Shamay-Tsoory, et al., "Intranasal Administration of Oxytocin Increases Envy and Schadenfreude (Gloating)," Biological Psychiatry, Vol. 66, No. 9, 2009, pp. 864-870. doi:10.1016/j.biopsych.2009.06.009

[8] A. H. Kemp and A. J. Guastella, “Oxytocin: Prosocial Behavior, Social Salience, or Approach-Related Behavior?” Biological Psychiatry, Vol. 67, No. 6, 2010, pp. e33-34. doi:10.1016/j.biopsych.2009.11.019

[9] J. A. Bartz, et al., "Oxytocin Selectively Improves Empathic Accuracy,” Psychological Science, Vol. 21, No. 10, 2010, pp. 1426-1428. doi:10.1177/0956797610383439

[10] G. Domes, M. Heinrichs, A. Michel, C. Berger and S. Herpertz, “Oxytocin Improves 'Mind-Reading' in $\mathrm{Hu}-$ mans,” Biological Psychiatry, Vol. 61 No. 6, 2007, pp. 731-733. doi:10.1016/j.biopsych.2006.07.015

[11] A. Guastella, P. Mitchell and M. Dadds, "Oxytocin Increases Gaze to the Eye Region of Human Faces,” Biological Psychiatry, Vol. 63, No. 1, 2008, pp. 3-5. doi:10.1016/j.biopsych.2007.06.026

[12] A. J. Guastella, et al., "Intranasal Oxytocin Improves Emotion Recognition for Youth with Autism Spectrum Disorders,” Biological Psychiatry, Vol. 67, No. 7, 2010, pp. 692-694. doi:10.1016/j.biopsych.2009.09.020

[13] E. Hollander, et al., "Oxytocin Increases Retention of Social Cognition in Autism,” Biological Psychiatry, Vol. 61, No. 4, 2007, pp. 498-503. doi:10.1016/j.biopsych.2006.05.030

[14] J. Green and E. Hollander, “Autism and Oxytocin: New Developments in Translational Approaches to Therapeutics," Neurotherapeutics, Vol. 7, No. 3, 2010, pp. 250257. doi:10.1016/j.nurt.2010.05.006

[15] Y. Chen, A. Ehlers, D. Clark and W. Mansell, "Patients with Generalized Social Phobia Direct Their Attention Away from Faces,” Behaviour Research Therapy, Vol. 40, 
No. 6, 2002, pp. 677-687. doi:10.1016/S0005-7967(01)00086-9

[16] J. C. Rohner, "The Time-Course of Visual Threat Processing: High Trait Anxious Individuals Eventually Avert Their Gaze from Angry Faces,” Cognition \& Emotion, Vol. 16, No. 6, 2002, pp. 837-844. doi:10.1080/02699930143000572

[17] S. Evans, S. S. Shergill and B. B. Averbeck, "Oxytocin Decreases Aversion to Angry Faces in an Associative Learning Task," Neuropsychopharmacology, Vol. 35, No. 13, 2010, pp. 2502-2509. doi:10.1038/npp.2010.123

[18] I. Labuschagne, et al., “Oxytocin Attenuates Amygdala Reactivity to Fear in Generalized Social Anxiety Disorder," Neuropsychopharmacology, Vol. 35, No. 12, 2010, pp. 2403-2413. doi:10.1016/j.biopsych.2007.06.026

[19] A. Guastella, P. Mitchell and M. Dadds, "Oxytocin Increases Gaze to the Eye Region of Human Faces,” Biological Psychiatry, Vol. 63, No. 1, 2008, pp. 3-5. doi:10.1016/j.biopsych.2007.06.026

[20] K. M. Dalton, et al., "Gaze Fixation and the Neural Circuitry of Face Processing in Autism," Nature Neuroscience, Vol. 8, No. 4, 2005, pp. 519-526. doi:10.1038/nn1421

[21] E. Andari, et al., "Promoting Social Behavior with Oxytocin in High-Functioning Autism Spectrum Disorders," Proceedings of the National Academy of Sciences USA, Vol. 107, No. 9, 2010, pp. 4389-4394. doi.org/10.1073/pnas.0910249107

[22] M. Fischer-Shofty, S. G. Shamay-Tsoory, H. Harari and Y. Levkovitz, "The Effect of Intranasal Administration of Oxytocin on Fear Recognition,” Neuropsychologia, Vol. 48, No. 1, 2010, pp. 179-184. doi:10.1016/j.neuropsychologia.2009.09.003

[23] E. Savaskan, R. Ehrhardt, A. Schulz, M. Walter and H. Schachinger, "Post-Learning Intranasal Oxytocin Modulates Human Memory for Facial Identity,” Psychoneuroendocrinology, Vol. 33, No. 3, 2008, pp. 368-374. doi:10.1016/j.psyneuen.2007.12.004

[24] P. Kirsch, et al., "Oxytocin Modulates Neural Circuitry for Social Cognition and Fear in Humans,” Journal of Neuroscience, Vol. 25, No. 49, 2005, pp. 11489-11493. doi:10.1523/JNEUROSCI.3984-05.2005

[25] D. Huber, P. Veinante and R. Stoop, "Vasopressin and Oxytocin Excite Distinct Neuronal Populations in the Central Amygdala,” Science, Vol. 308, No. 5719, 2005, pp. 245-248. doi:10.1126/science.1105636

[26] R. Adolphs and M. Spezio, "Role of the Amygdala in Processing Visual Social Stimuli," Progress in Brain Research, Vol. 156, 2006, pp. 363-378. doi:10.1016/S0079-6123(06)56020-0

[27] J. Haxby, E. Hoffman and M. Gobbini, "Human Neural Systems for Face Recognition and Social Communication,” Biological Psychiatry, Vol. 51, No. 1, 2002, pp. 5967. doi:10.1016/S0006-3223(01)01330-0

[28] P. Vuilleumier and G. Pourtois, "Distributed and Interactive Brain Mechanisms during Emotion Face Perception: Evidence from Functional Neuroimaging," Neuropsychologia, No. 45 No. 1, 2007, pp. 174-194. doi:10.1016/j.neuropsychologia.2006.06.003

[29] J. S. Morris, et al., “A Neuromodulatory Role for the Human Amygdala in Processing Emotional Facial Expressions,” Brain Cognition, Vol. 121 No. 1, 1998, pp. 47-57. doi: 10.1093/brain/121.1.47

[30] G. Domes, M. Heinrichs, J. Gläscher, C. Büchel, D. Braus and S. Herpertz, "Oxytocin Attenuates Amygdala Responses to Emotional Faces Regardless of Valence," Biological Psychiatry, Vol. 62, No.10, 2007, pp. 11871190. doi:10.1016/j.biopsych.2007.03.025

[31] P. Petrovic, R. Kalisch, T. Singer and R. J. Dolan, “Oxytocin Attenuates Affective Evaluations of Conditioned Faces and Amygdala Activity,” Journal of Neuroscience, Vol. 28, No. 26, 2008, pp. 6607-6615. doi:10.1523/JNEUROSCI.4572-07.2008

[32] G. Domes, et al., "Effects of Intranasal Oxytocin on Emotional Face Processing in Women," Psychoneuroendocrinology, Vol. 35, No. 1, 2010, pp. 83-93. doi:10.1016/j.psyneuen.2009.06.016

[33] J. A. Bartz, J. Zaki, N. Bolger and K. Ochsner, "Social Effects of Oxytocin in Humans: Context and Person Matter," Trends in Cognitive Sciences, Vol. 15, No. 7, pp. 301-309. doi: 10.1016/j.tics.2011.05.002

[34] M. Gamer, B. Zurowski and C. Büchel, "Different Amygdala Subregions Mediate Valence-Related and Attentional Effects of Oxytocin in Humans," Proceedings of the National Academy of Sciences USA, Vol. 107, No. 2, 2010, pp. 9400-9405. doi:10.1073/pnas.1000985107

[35] T. Kimura, O. Tanizawa, K. Mori, M. J. Brownstein and H. Okayama, "Structure and Expression of a Human Oxytocin Receptor,” Nature, Vol. 356, No. 6369, 1992, pp. 526-529. doi:10.1038/356526a0

[36] S. Jacob, C. W. Brune, C. S. Carter, B. L. Leventhal, C. Lord and E. H. Cook Jr., "Association of the Oxytocin Receptor Gene (OXTR) in Caucasian Children and Adolescents with Autism,” Neuroscience Letters, Vol. 417, No. 1, 2007, pp. 6-9. doi:10.1016/j.neulet.2007.02.001

[37] E. Lerer, S. Levi, S. Salomon, A. Darvasi, N. Yirmiya and R. P. Ebstein, "Association between the Oxytocin Receptor (OXTR) Gene and Autism: Relationship to Vineland Adaptive Behavior Scales and Cognition,” Molecular Psychiatry, Vol. 13, No. 10, 2007, pp. 980-988. doi:10.1038/sj.mp.4002087

[38] S. Wu, et al., "Positive Association of the Oxytocin Receptor Gene (OXTR) with Autism in the Chinese Han Population,” Biological Psychiatry, Vol. 58, No. 1, 2005, pp. 74-77. doi:10.1016/j.biopsych.2005.03.013

[39] A. K. Wermter, I. Kamp-Becker, P. Hesse, G. SchulteKörne, K. Strauch and H. Remschmidt, "Evidence for the Involvement of Genetic Variation in the Oxytocin Receptor Gene (OXTR) in the Etiology of Autistic Disorders on High-Functioning Level," American Journal of Medical Genetics Part B: Neuropsychiatric Genetics, Vol. 153B, No. 2, 2009, pp. 629-639. doi:10.1002/ajmg.b.31032

[40] X. Liu, et al., "Association of the Oxytocin Receptor (OXTR) Gene Polymorphisms with Autism Spectrum Disorder (ASD) in the Japanese Population,” Journal of Human Genetics, Vol. 55, No. 3, 2010, pp. 137-141. 
doi:10.1038/jhg.2009.140

[41] C. Yrigollen, et al., "Genes Controlling Affiliative Behavior as Candidate Genes for Autism,” Biological Psychiatry, Vol. 63, No. 10, 2008, pp. 911-916. doi:10.1016/j.biopsych.2007.11.015

[42] K. E. Tansey, et al., "Oxytocin Receptor (OXTR) Does Not Play a Major Role in the Aetiology of Autism: Genetic and Molecular Studies,” Neuroscience Letters, Vol. 474, No. 3, 2010, pp. 163-167. doi:10.1016/j.neulet.2010.03.035

[43] S. Israel, et al., "The Oxytocin Receptor (OXTR) Contributes to Prosocial Fund Allocations in the Dictator Game and the Social Value Orientations Task," PLoS ONE, Vol. 4, No. 5, 2009, p. e5535. doi:10.1371/journal.pone.0005535

[44] M. J. Bakermans-Kranenburg and M. H. van Ijzendoorn, "Oxytocin Receptor (OXTR) and Serotonin Transporter (5-HTT) Genes Associated with Observed Parenting," Social Cognitive and Affective Neuroscience, Vol. 3, No. 2, 2008, pp. 128-134. doi:10.1093/scan/nsn004

[45] S. M. Rodrigues, L. R. Saslow, N. Garcia, O. P. John and D. Keltner, "Oxytocin Receptor Genetic Variation Relates to Empathy and Stress Reactivity in Humans," Proceedings of the National Academy of Sciences USA, Vol. 106, No. 50, 2009, pp. 21437-21441. doi:10.1073/pnas.0909579106

[46] H. Tost, et al., "A common Allele in the Oxytocin Receptor Gene $(O X T R)$ Impacts Prosocial Temperament and Human Hypothalamic-Limbic Structure and Function,” Proceedings of the National Academy of Sciences USA, Vol. 107, No. 31, 2010, 13936-13941. doi:10.1073/pnas.1003296107

[47] H. Inoue, H. Yamasue, M. Tochigi, O. Abe, X. Liu, Y. Kawamura, et al., "Association between the Oxytocin Receptor Gene and Amygdalar Volume in Healthy Adults," Biological Psychiatry, Vol. 68, No. 11, 2010, pp. 10661072. doi:10.1016/j.biopsych.2010.07.019

[48] D. J. Furman, M. C. Chen and I. H. Gotlib, "Variant in Oxytocin Receptor Gene is Associated with Amygdala Volume,” Psychoneuroendocrinology, Vol. 36, No. 6, 2011, pp. 891-897. doi:10.1016/j.psyneuen.2010.12.004

[49] C. Montag, C. J. Fiebach, P. Kirsch and M. Reuter, "In- teraction of 5-HTTLPR and a Variation on the Oxytocin Receptor Gene Influences Negative Emotionality,” Biological Psychiatry, Vol. 69, No. 6, 2011, pp. 601-603. doi:10.1016/j.biopsych.2010.10.026

[50] H. E. Nelson, “National Adult Reading Test,” 2nd Edition, NFER-Nelson, Windsor, 1991.

[51] J. C. Barrett, B. Fry, J. Maller and M. J. Daly, "Haploview: Analysis and Visualization of LD and Haplotype Maps,” Bioinfomatics, Vol. 21, No. 2, 2005, pp. 263-265. doi:10.1093/bioinformatics/bth457

[52] H. C. Breiter, et al., "Response and Habituation of Human Amygdala during Visual Processing of Facial Expression,” Neuron, Vol. 17, No. 5, 1996, pp. 875-887. doi:10.1016/S0896-6273(00)80219-6

[53] J. E. LeDoux, “The Emotional Brain," Simon and Shuster, New York, 1996.

[54] L. Pessoa, M. McKenna, E. Gutierrez and L. G. Ungerleider, "Neural Processing of Emotional Faces Requires Attention," Proceedings of the National Academy of Sciences USA, Vol. 99, No. 17, 2002, pp. 11458-11463. doi:10.1073/pnas.172403899

[55] R. T. Schultz, "Developmental Deficits in Social Perception in Autism: The Role of the Amygdala and Fusiform Face Area,” International Journal of Developmental Neuroscience, Vol. 23, No. 2-3, 2005, pp. 125-141. doi:10.1016/j.ijdevneu.2004.12.012

[56] K. Pierce, R. A. Müller, A. G. Allen and E. Courchesne, "Face Processing Occurs outside the Fusiform 'Face Area' in Autism: Evidence from fMRI,” Brain, Vol. 124, No. 10, 2001, pp. 2059-2037. doi:10.1093/brain/124.10.2059

[57] F. Champagne, D. Francis, A. Mar and M. Meaney, "Variations in Maternal Care in the Rat as a Mediating Influence for the Effects of Environment on Development,” Physiology \& Behavior, Vol. 79, No. 3, 2003, pp. 359-371. doi:10.1016/S0031-9384(03)00149-5

[58] H. S. Kim, et al., "Culture, Distress, and Oxytocin Receptor Polymorphism (OXTR) Interact to Influence Emotional Support Seeking," Proceedings of the National Academy of Sciences USA, Vol. 107, No. 36, 2010, pp. 15717-15721. doi:10.1073/pnas.1010830107 